\title{
The effects of response mode and stimulus laterality on reaction time in a Sternberg task
}

\author{
MICHELLE A. ADKINS, W. A. HILLIX, and JAMES W. BROWN \\ San Diego State University, San Diego, California
}

\begin{abstract}
The effects of visual field (right or left), mode of presentation (unilateral or bilateral), and response mode (manual or verbal) on reaction time were investigated in a Sternberg task. Subjects responded with their left hands, with their right hands, or verbally to indicate whether a stimulus was in or out of a memory set. Unilateral presentation produced significantly shorter reaction times than bilateral presentation did $(p<.001)$. Stimuli in the memory set were responded to more quickly $(p<.001)$ than were those out of the memory set. Presentation mode interacted with visual field $(p<.01)$. Reaction times to left visual field stimuli were significantly shorter only when stimuli were presented bilaterally. Response mode interacted with memory set $(p<.001)$. Reaction times for letters in the memory set were faster than those for letters out of the memory set for manual but not for verbal responses. These results pose problems for interpreting memory set and divided visual field studies.
\end{abstract}

Divided visual field studies of reaction time to lateralized stimuli have provided important but often confusing information about the functions of and differences between the cerebral hemispheres. If subjects in such experiments respond manually to a stimulus and then state verbally what stimulus has been presented, they can respond immediately upon detecting the stimulus, and decide later what stimulus was presented. Reaction time may then reflect only the time to detect the stimulus, and not reveal differences in hemispheric processing when they exist. In order to avoid this difficulty, several experimenters (e.g., Klatzky \& Atkinson, 1971) have used a Sternbergtype memory set task. In this task, subjects must process the stimulus information prior to responding, in order to decide whether a probe stimulus is in or out of a memory set. When subjects are asked to indicate as quickly as possible whether target items are in or out of the set, reaction times generally increase linearly with the number of items in the memory set, regardless of whether probe stimuli are in or out of the set (Sternberg, 1966, 1969). In most experiments, slopes are identical for negative and positive responses. This result is usually interpreted as evidence that subjects scan all items in the memory set before categorizing the probe stimulus (exhaustive scanning, serial comparison), rather than stop when a match between the probe and an item in the memory set is found (self-terminating search).

Cohen (1973) did a related experiment, in which he used stimulus sets rather than memory sets. He presented two, three, or four letters in the left or right visual field via a tachistoscope, and subjects judged whether all of the letters were identical. Reaction times increased as the

Correspondence should be addressed to W. A. Hillix, Department of Psychology, San Diego State University, San Diego, CA 92182-0350. number of letters in the set increased for stimuli presented to the left hemisphere, as was expected from the exhaustive scanning theory. However, reaction time did not increase as the number of items increased for stimuli presented to the right hemisphere, indicating that the right hemisphere was performing parallel processing. Klatzky and Atkinson (1971) also argued that their results indicated hemispheric differences in processing capabilities; the right hemisphere appeared to be better at comparing the shapes of letters, but left-hemisphere capability was required for naming pictures and extracting the first letters of the names. Thus there is evidence that the hemispheres process information differently in both perception and memory.

Boles (1987) found that bilateral presentation revealed hemispheric differences much larger than those found with unilateral presentation. Judgments about bar graphs produced a left visual field advantage, and judgments about letters produced a right visual field advantage. Right visual field superiority has been found in numerous experiments in which linguistic stimuli were used (Schmuller, 1979; Segalowitz \& Stewart, 1979). Many investigators believe that letters, words, and digits are recognized better in the right visual field (Zaidel, 1985) and that spatial stimuli are recognized better in the left visual field (Gross, 1972).

However, this "agreement" is far from universal. Yund, Efron, and Nichols (1990) consistently find that spatial stimuli are recognized more accurately in the right visual field, whether or not stimuli are simultaneously presented in the opposite field. They account for the right visual field superiority via a serial scanning mechanism that tends to scan the right field first. This biased scanner would operate when subjects did not know in advance where the critical stimuli would appear. On the other hand, Haun (1978) found left visual field superiority for both geometric forms and letters. Davis and Schmit (1973) 
concluded that visually identical letters might be matched better when presented in the left visual field. Bradshaw, Hicks, and Rose (1979) found a left visual field advantage for briefly presented stimuli.

Some of the conflicting results of visual field studies may be accounted for by differences in procedure, in particular by differences in mode of response and mode of stimulus presentation. Most experimenters assume that response mode is relatively independent of other variables (Beaumont, 1982). Left- and right-hand responses are often used equally throughout an experiment, but some experimenters find it unnecessary to vary the response mode (Cohen, 1972). Other experimenters have used bimanual responses (Bradshaw, Bradley, Gates, \& Patterson, 1977). No general difference between right- and left-hand manual responses has been discovered, but the interaction of response mode with other variables seems not to have been studied systematically. Response mode could affect processing strategy, and it is therefore important to examine verbal and manual responding within the same set of experimental conditions.

The present study was designed to provide reliable baseline information about the effects of some methodological variables involved in lateralization research by using the Sternberg task. Left-hand, right-hand, and verbal responses were all used.

\section{METHOD}

\section{Subjects}

Twenty-four male and 24 female introductory psychology students from San Diego State University received class credit for participation. All subjects were right-handed and had 20/20 eyesight or eyesight corrected to 20/20. A 3 (response mode) $\times 2$ (unilateral or bilateral probe) $\times 2$ (in or out of memory set) $\times 2$ (visual field) $\times 2$ (response assignment) mixed design was used, with response assignment the only between-subject variable. The order in which the three response modes were presented was counterbalanced across subjects.

Unilateral or bilateral stimulus presentation, visual field (left or right) to which the subject responded, memory set (in or out), and response mode (left hand, right hand, or verbal) were all manipulated within subjects. Response assignment (middle or index finger response indicating that the probe was in the memory set) was varied between subjects to avoid confusing the subjects and to eliminate any possible confounding of practice with response assignment.

\section{Materials and Apparatus}

Each subject was given a handedness questionnaire, to ensure strong right-handed tendencies. A computer-controlled video screen (oscilloscope) was used to present data. The subjects responded in the verbal response condition by using a microphone that activated a voice key, and they responded under the manual conditions by using a panel with two keys. "In" and "out" were used as the verbal responses rather than "yes" and "no" because we believed that the rise times for "in" and "out" would be more similar and thus activate the voice key at more nearly identical times after the initiation of the verbal response. An IBM-AT-compatible computer system presented the stimuli and recorded response times with millisecond accuracy.

\section{Procedure}

Subjects signed up individually for 45-min sessions. Each subject received one of the six counterbalanced orders of the three response modes. The subjects first filled out a handedness questionnaire and signed a consent form. Next, the first 24 subjects were given instructions that read, in part:
In each condition, you will be responding to letters as being either in or out of a three-letter memory set (the memory set being $\mathrm{H}, \mathrm{Y}, \mathrm{M}$ ). Letters will flash on one or both sides of the screen with an arrow pointing in the direction of the letter you should respond to.... I will tell you what response mode to use for each section. When responding with either hand you will use two buttons (an "in" button and an "out" button); your index finger will always be "in" and your middle finger will always be "out." When you are answering verbally, you merely say "in" or "out" loudly into the microphone. Simply look at the center $(+)$ fixation point and answer appropriately.

The second 24 subjects received the same instructions, except that they were told to respond "in" with the middle finger and "out" with the index finger. The subjects were not informed of the identities of the letters (X, I, V, A, T) that were "out" of the memory set.

Subjects were then free to ask questions, and 24 practice trials were given. If a subject's performance during the practice session showed that he or she did not understand the directions, the necessary directions were repeated orally until the subject understood. Ninety-six trials were given, 32 in each response mode. Four trials were run for each of the 24 possible variable combinations. Directly before the real trials began, the subjects were told to respond to the stimuli as quickly as possible while remaining accurate. If incorrect responses were made, the stimuli were presented again until all responses were correct. No additional directions were given once the real trials were in progress. After each set of trials with a given response mode, the subjects were given a chance to rest their eyes and were told they were "doing great."

Each trial began with a 100 -msec blank period. This was followed by a " +" fixation point presented at the center of the screen for a random duration between 1,000 and $2,000 \mathrm{msec}$. The fixation point changed to an arrow (" $<$ " or " > ") for $100 \mathrm{msec}$ before the probe letter was presented. Probe letters were on the screen for $100 \mathrm{msec} 2^{\circ}$ to the left or right of the focal point. The intertrial interval was $3,000 \mathrm{msec}$. Trials were treated as errors and repeated if the response time was less than $100 \mathrm{msec}$ or more than $5,000 \mathrm{msec}$. All repeated trials occurred at the end of the trials for each particular response mode. Thus all reaction times are for correct trials, although presumably some trials were correct by chance.

Reaction time was automatically recorded from the start of the stimulus presentation until a button was depressed or until the voice key was activated, depending on the response mode. The experimenter recorded whether verbal responses were correct or incorrect by pressing the appropriate key on the computer keyboard. When subjects were finished, they were told the purpose of the experiment.

\section{RESULTS}

A 3 (response mode) $\times 2$ (laterality) $\times 2$ (visual field) $\times 2$ (memory set) $\times 2$ (response assignment) mixed design analysis of variance was performed on the data. A significant main effect was found for laterality $[F(1,46)=$ $86.89, p<.001$ ]; reaction times were significantly faster when stimuli were presented unilaterally $(M=866.99)$ than when they were presented bilaterally $(M=989.36)$.

The main effect for memory set was also significant $[F(1,46)=26.27, p<.001]$. Reaction times were significantly shorter when subjects were responding to letters in the memory set $(M=895.24)$ than they were when subjects were responding to letters out of the memory set $(M=961.1)$.

An interaction was found between visual field and universus bilateral presentation $[F(1,46)=9.55, p<.01]$. A Tukey test indicated that, in the bilateral condition, subjects responded faster to stimuli in the left visual field $(M=971.80)$ than to stimuli in the right visual field $(M=1,006.93)$. Figure 1 shows the mean reaction time for stimuli presented in the left or right visual field either unilaterally or bilaterally. 


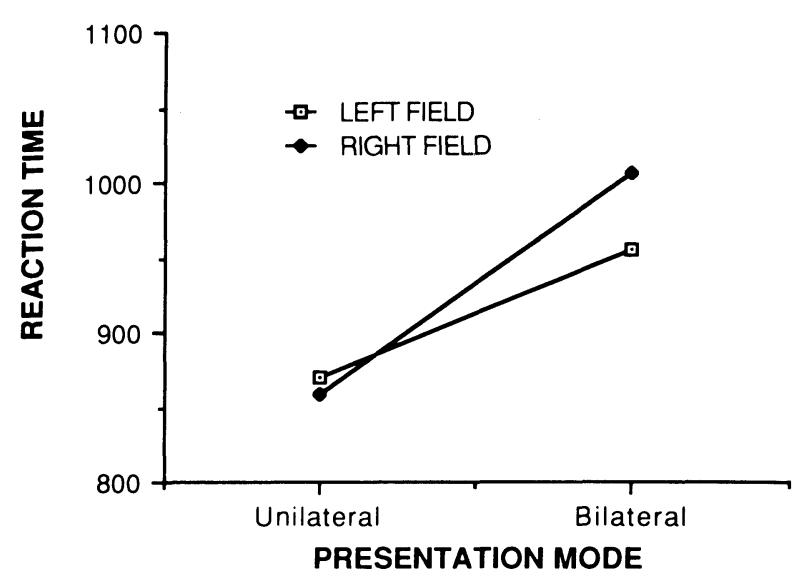

Figure 1. Mean reaction time in milliseconds for stimuli presented in the left or right visual field either unilaterally or bilaterally.

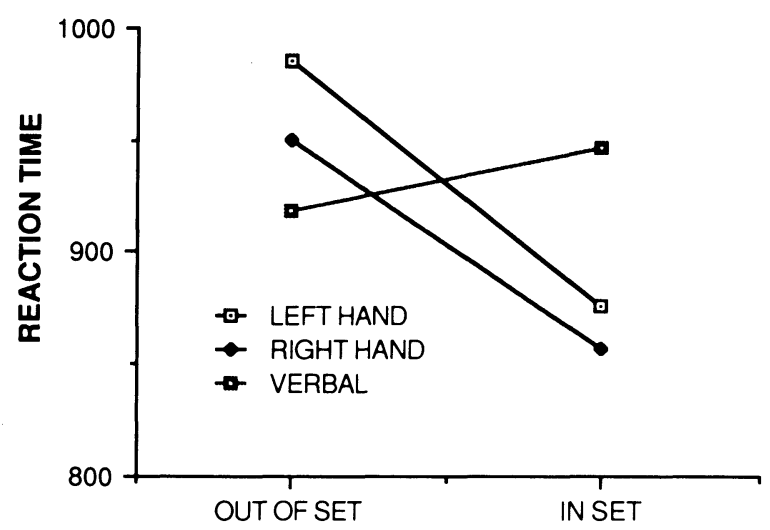

IN/OUT MEMORY SET

Figure 2. Mean reaction time in milliseconds for right-hand, lefthond, or verbal responses to stimuli either in or out of the memory set.

The interaction between response mode and memory set was also significant $[F(2,92)=18.75, p<.001]$. Figure 2 shows the nature of this interaction. Tukey tests showed that several differences were significant. When stimuli were in the memory set and subjects responded manually either with the left or with the right hand, reaction times were significantly shorter than for any of the other conditions.

\section{DISCUSSION}

The significant interactions demonstrate the lack of generality of the two significant main effects; that is, there were effects of visual field only for bilaterally presented stimuli, and responses to items in the memory set were faster than those to items out of the memory set only when manual responses were used.

Thus our "linguistic" materials were sometimes processed faster in the right hemisphere; when responding manually, subjects probably responded to our single letters as shapes, for which the right hemisphere often produces reaction times equal to or shorter than those produced by the left hemisphere (such as in Haun, 1978; Klatzky \& Atkinson, 1971). The occurrence of differences may well depend on the match- ing strategy adopted, with a particular type of material treated as "linguistic" at one time and not at another.

Reaction times were equal for letters in and out of the memory set when responses were verbal, but they were significantly longer for items out of the memory set when responses were manual. The usual finding of different reaction times for "in" and "out" may be related to the fact that manual responses are used more often (see, e.g., Bradshaw et al., 1977; Eriksen, Eriksen, \& Hoffman, 1986; Muthig \& Ulrich, 1985; Orenstein \& Hamilton, 1977; Strayer, Wickens, \& Braune, 1987).

Our results might suggest that a self-terminating search was being used when responses were manual, but many studies indicate that processing in this task is serial and exhaustive (cf. Orenstein \& Hamilton, 1977; Sternberg, 1966, 1969). An additional problem with this explanation is that it should apply when verbal as well as manual responses are required, and the equality of the verbal reaction times for probes in and out of the memory set suggests that an exhaustive search strategy was used when responses were verbal.

Although an exhaustive search is indicated in most cases, "out" responses often take longer than "in" responses, regardless of the number of items searched; that is, the intercept of the line expressing the relationship between number of items and reaction time is higher for "out" than for "in." Nevertheless, the present results for verbal responses demonstrate that "out" does not always take longer than "in."

Subjects are more likely to code the probes and memory set acoustically when they are required to make a verbal response than when they are required to make a manual response. Acoustic coding may impose a heavier processing load that interferes with some aspect of processing probes in the memory set, but that does not change the processing of probes that are not in the memory set.

A possible explanation is that probes that are in the memory set usually can be "recognized" or "loaded" faster than probes out of the set, which was not possible under the verbal response conditions of the present experiment. This explanation is consistent with the present data, with previous results, and with some models of the process. If probes in a memory set could be recognized via template matching, but probes out of the set required feature analysis, a reaction time advantage might accrue to in-set probes. Such a difference in recognition strategy might be eliminated by the verbal response requirement.

We were surprised at the near-identical left- and right-hand reaction time patterns, both of which differed markedly from the verbal response pattern. The similarity of left- and right-hand patterns is, however, consistent with the results of Yund et al. (1990) and with the results of others who have used both error and reaction time measures with manual responses as indicants.

Yund et al. (1990) believe that preferential scanning of the right visual field could mask, or even reverse, right-hemisphere superiority when it exists. In their experiments, subjects do not know the visual field in which stimuli will appear (unilateral presentation) or in which field the probe stimulus will appear (bilateral presentation). In our experiment, a " > " or " <" sign appeared for $100 \mathrm{msec}$ prior to the presentation of the letter to which the subject was to respond. The stimulus thus controlled the scanning mechanism, and responses to stimuli in the left visual field were faster than responses to stimuli in the right visual field when stimuli were presented bilaterally, reversing the findings obtained by Yund et al. with the use of similar stimuli but with a different scanning task and no control of scanning.

Our experiment involved several simple independent variables because we wanted to examine the effects of basic methodological variables on reaction time. We conclude that hand and finger of response do not affect reaction time, but that verbal responses may yield results quite different from those produced by manual responses made with either hand. Our results confirm that the bilateral presentation of stimuli produces larger hemispheric differences than does unilateral presentation, even when the putative scanning mechanism is directed to the relevant visual field.

The interaction between response mode and set membership indicates that experimenters using the memory set paradigm must generalize very cautiously if their experiments have not included both verbal and manual responding, and that experimenters in lateralization must pay attention to the modes of both stimulus presentation and response. Variations in either may affect the strategies subjects use to solve whatever problems are presented, and there is a danger that the differences observed will 
be interpreted as hemispheric differences when they are really attributable to side-biased strategies. Despite the long history of divided visual field research, we are just beginning to learn which methodological variables (such as hand of response and assignment of responses to fingers) seem not to interact with other variables and which ones do (whether stimuli are unilateral or bilateral, or whether subjects are directed to the critical visual field either by a warning stimulus or by consistent presentation to one side or the other, or whether responding is manual or verbal).

\section{REFERENCES}

Beaumont, J. G. (1982). Studies with verbal stimuli. In J. G. Beaumont (Ed.), Divided visual field studies of cerebral organisation (pp. 57-86). London: Academic Press.

BOLEs, D. B. (1987). Reaction time asymmetry through bilateral versus unilateral stimulus presentation. Brain \& Cognition, 6, 321-333.

Bradshaw, G. J., Hicks, R. E., \& Rose, B. (1979). Lexical discrimination and letter-string identification in the two visual fields. Brain \& Language, 8, 10-18.

Bradshaw, J. L., Bradley, D., Gates, A., \& Patterson, K. (1977). Serial, parallel, or holistic identification of single words in the two visual fields? Perception \& Psychophysics, 21, 431-438.

CoHEN, G. (1972). Hemispheric differences in a letter classification task. Perception \& Psychophysics, 11, 139-142.

CoHEN, G. (1973). Hemispheric differences in serial versus parallel processing. Journal of Experimental Psychology, 97, 349-356.

DAvis, R., Schmit, V. (1973). Visual and verbal coding in the interhemispheric transfer of information. Acta Psychologica, 37, 229-240.

Eriksen, B. A., Eriksen, C. W., \& Hoffman, J. E. (1986). Recognition memory and attentional selection: Serial scanning is not enough. Journal of Experimental Psychology: Human Perception \& Performance, 12, 476-483.

Gross, M. M. (1972). Hemispheric specialization for processing of visually presented verbal and spatial stimuli. Perception \& Psychophysics, 12, 357-363.

HAUN, F. (1978). Functional dissociation of the hemispheres using foveal visual input. Neuropsychologia, 16, 725-733.

Klatzky, R. L., \& AtKinson, R. C. (1971). Specialization of the cerebral hemispheres in scanning for information in short-term memory. Perception \& Psychophysics, 10, 335-338.

MUThig, K.-P., \& UlRiCh, R. (1985). Selective search in short-term memory under ideal conditions of test stimulus categorization. Memory \& Cognition, 13, 29-36.

Orenstein, H. B., Hamilton, K. M. (1977). Memory load, critical features and retrieval processes in facial recognition. Perceptual \& Motor Skills, 45, 1079-1087.

SCHMUller, J. (1979). Hemispheric asymmetry for alphabetic identification: Scaling analysis. Brain \& Language, 8, 263-274.

Segalowitz, S. J., \& Stewart, C. (1979). Left and right lateralisation or letter matching: Strategy and sex differences. Neuropsychologia, 17, 521-525.

STERNBERG, S. (1966). High-speed scanning in human memory. Science, $153,652-654$.

STERNBerG, S. (1969). Memory-scanning: Mental processes revealed by reaction-time experiments. American Scientist, 4, 421-457.

Strayer, D. L., Wickens, C. D., \& Braune, R. (1987). Adult age differences in the speed and capacity of information processing: 2 . An electrophysiological approach. Psychology \& Aging, 2, 99-110.

YuND, E. W., EFRON, R., Nichols, D. R. (1990). Target detection in one visual field in the presence or absence of stimuli in the contralateral field by right- and left-handed subjects. Brain \& Cognition, 12, 117-127.

ZAIDEL, D. W. (1985). Hemifield tachistoscopic presentations and hemispheric specialization in normal subjects. In F. D. Benson \& E. Zaidel (Eds.), The dual brain (pp. 143-155). New York: Guilford.

(Manuscript received August 5, 1991.) 